\title{
Author Correction: Therapeutic implications of germline genetic findings in cancer
}

Subotheni Thavaneswaran, Emma Rath, Kathy Tucker, Anthony M. Joshua, Dominique Hess, Mark Pinese (D), Mandy L. Ballinger and David M. Thomas

Nature Reviews Clinical Oncology (2019) https://doi.org/10.1038/s41571-019-0179-3

Published online 19 February 2019

The originally published article contained errors in the main text and in figure 1 in the reported number of patients with pathogenic or likely pathogenic germline variants. The originally reported numbers did not take into account the presence of more than one variant in an individual patient. This has been corrected in the HTML and PDF versions of the manuscript.

https://doi.org/10.1038/s41571-019-0212-6 I Published online 17 April 2019 